Neben einer theoretischen Einordnung der Öffentlichkeits- und Gegenöffentlichkeitsbegriffe wird das Thema empirisch anhand von vier Forschungsfragen bearbeitet. Dabei wird im Rahmen von Forschungsfrage 1 untersucht, welche alternativen Nachrichtenmedien im DACH-Raum aktuell online via Website und Accounts auf Digitalplattformen agieren. Forschungsfrage 2 legt den Fokus auf das Selbstverständnis alternativer Nachrichtenmedien, um diese zu definieren und typologisch einzuordnen. Forschungsfrage 3 widmet sich schliesslich einer netzwerkanalytischen Betrachtung alternativer und professioneller deutschsprachiger Nachrichtenmedien auf der Plattform Twitter. Dieser deskriptive Zwischenschritt ist massgeblich für die Beantwortung von Forschungsfrage 4, im Zuge derer jene Netzwerkrelationen interpretativ analysiert werden.

\title{
I.2 Relevanz
}

Was passiert, wenn sich in Zeiten digitaler Kommunikation der erweiterte Kreis an Kommunikator_innen oder «pseudojournalistischen» Anbietern (Eisenegger, 2017) nicht an journalistische Standards, wie beispielsweise Quellentransparenz oder Objektivität, halten? Wenn desinformative, verschwörungstheoretische Beiträge öffentlich zirkulieren? Oder politisch gefärbte, polarisierende Meinungen und Nachrichten verbreitet werden? Die Auseinandersetzung mit alternativen Nachrichtenmedien ruft ebensolche Fragen hervor. Die Relevanz, alternative Nachrichtenmedien im deutschsprachigen Raum $\mathrm{zu}$ untersuchen, ergibt sich unbestreitbar aufgrund potentieller Gefahren für die soziale Ordnung respektive Demokratie. Die von der Etablierung digitaler Plattformen geprägte digitale Öffentlichkeit bietet aufgrund ihres partizipativen Charakters den idealen Nährboden für alternative Kommunikator_innen. Ein auf Vernunft und Argumenten basierter Diskurs als Basis unserer Demokratie ist bei alternativen Kommunikator_innen aber gerade dann in Frage zu stellen, wenn absichtlich Sachverhalte verdreht, Falschnachrichten verbreitet, oder Gruppen diffamiert werden. Ausserdem impliziert der Begriff «Alternativmedium» eine relationale Abgrenzung zu klassischen Medien. Sie positionieren sich gegen die mediale und politische Öffentlichkeit, wodurch einerseits die Gefahr besteht, dass sich Publika von Alternativmedien ein Bild der Wirklichkeit in abgeschlossenen Teil- respektive Gegenöffentlichkeiten machen und mit professionellen journalistischen Beiträgen kaum oder gar nicht in Berührung kommen. Darüber hinaus können alternative Nachrichtenmedien eine Polarisierung der Gesellschaft weiter fördern - im Sinne von: Wir gegen die anderen. Nichtsdestoweniger erscheint es wesentlich, das Spektrum alternativer Nachrichtenmedien genauer unter die Lupe zu nehmen. Eine dichotome Unterscheidung in Öffentlichkeit und Gegenöffentlichkeit - respektive: professionelle und alternative Nachrichtenmedien - erscheint vor allem 
in der digitalen Öffentlichkeit mit einer Vielzahl an Kommunikator_innen fraglich. Bezugnehmend auf die Ursprünge alternativer Nachrichtenmedien wird es zudem als zu stark einschränkend erachtet, Alternativmedien per se als demokratiefeindlich und gefährlich einzustufen. Gesellschaftliche Implikationen können nur dann getroffen werden, wenn das Spektrum alternativer Nachrichtenmedien möglichst wertfrei und unvoreingenommen erforscht wird.

\section{I.3 Vorgehen}

Die vorliegende Arbeit bemüht sich darum, die Bedeutung von Online-Gegenöffentlichkeiten in Form alternativer Nachrichtenmedien im deutschsprachigen DACH-Raum aus einer phänomenologischen Perspektive explorativ zu erforschen. Neben einer multimethodischen Herangehensweise dient der theoretische Rahmen basierend auf Öffentlichkeits- und Gegenöffentlichkeitstheorien wie auch relational-soziologischen Ansätzen einer Einbettung des zu analysierenden Phänomens.

Dem Begriff der Öffentlichkeit widmet sich Kapitel II. Hierbei wird angestrebt, unterschiedliche Öffentlichkeitstheorien (Kapitel II.I) und den Strukturwandel der Öffentlichkeit zu skizzieren (Kapitel II.2) und in Anbetracht einer antizipierten digitalen Transformation (oder eines digitalen Strukturwandels) der Öffentlichkeit zu reflektieren (Kapitel II.3). Das Thema «Gegenöffentlichkeiten» wird in Kapitel III erörtert. Im Zuge dieses Kapitels wird neben einer obligatorischen Begriffsdefinition (Kapitel III.I) ein Schwerpunkt auf gesellschaftliche Bedingungen hinsichtlich der Etablierung von Gegenöffentlichkeiten gelegt. Dabei konzentriert sich Kapitel III.2 auf den Einfluss des sozialen Wandels und Kapitel III.3 auf den medialen Wandel. Das konkrete Untersuchungsphänomen dieser Arbeit - alternative Nachrichtenmedien als Gegenöffentlichkeiten - wird in Kapitel III.4 unter Einbindung des aktuellen Forschungsstandes zu den Themen «Desinformation» und «Verschwörung» theoretisch erarbeitet. Neben einer explorativen Untersuchung alternativer Nachrichtenmedien interessiert sich diese Studie insbesondere für deren Vernetzung im DACH-Raum, da von einem relationalen Verhältnis zwischen Öffentlichkeit und Gegenöffentlichkeit ausgegangen wird und die Logik der Vernetzung vor allem digitale Öffentlichkeiten massgeblich prägt. Theoretisch stützt sich diese Arbeit diesbezüglich auf die relationale Soziologie (Kapitel IV), die anhand klassischer soziologischer Schriften von Georg Simmel, Norbert Elias und Pierre Bourdieu aufgearbeitet wird (Kapitel IV.I). Diese theoretischen Ansätze werden aktuellen Forschungstrends hinsichtlich der empirischen Analyse von Netzwerken auf digitalen Plattformen gegenübergestellt (Kapitel IV.2). Nach einer theoretischen Zwischenbilanz und Darstellung der empirischen Forschungsfragen (Kapitel V) widmet sich Kapitel VI dem 